\title{
INDICIOS PARA LA RECONSTRUCCION DE CLASIFICADORES EN EL SINTAGMA NOMINAL PROTOCHIBCHA (1)
}

Adolfo Constenla Umaña

\begin{abstract}
Until now, there has not been any research specifically aimed at morphosyntactic reconstruction in the field of Chibchan linguistics, in which the studies following the comparative method have focused on phonology.

The purpose of this article is to contribute to the reconstruction of a piece of proto-Chibchan morphosyntax.

It is proposed here that two lexical items with the meaning of 'seed' and 'bone, stick' were used in that language as classifiers of referents with round and cylindric shapes, respectively.

Evidence from most of the languages of proven Chibchan affinities is offered in support of this hypothesis.
\end{abstract}

\section{INTRODUCCION}

Hasta el presente no se han dado investigaciones destinadas específicamente a la reconstrucción morfosintáctica en el campo de las lenguas de la familia chibcha y las de parentesco comprobado con ella.

Los estudios realizados empleando el método de inspección (por ejemplo, Beuchat y Rivet 1910, Rivet 1924, Conzemius 1929, Greenberg 1987) han hecho en, algunos casos, propuestas sobre la existencia de determinados morfemas y categorías gramaticales en el antepasado común, pero, por supuesto, sin aportar indicios suficientes y sistemáticos en favor de ellas ni intentar, por motivos obvios, reconstruir. Concretamente, en los casos en que el centro de atención fueron los elementos de valor clasificador - como en el del artículo de LéviStrauss (1948) sobre las lenguas chibchas y el nambicuara, por ejemplo- lo planteado se desvirtúa habitualmente por la falta de distinción entre las semejanzas de orden puramente tipológico y las verdaderamente pertinentes desde el punto de vista de la lingüística diacrónica.

Los trabajos que han efectuado reconstrucciones con base en el método comparativo (Wheeler 1972, Constenla Umaña 1981, Holt 1986) han incluido, entre los étimos propuestos, elementos de carácter gramatical, pero solo uno de ellos (Wheeler, ibidem) ha sugerido hipótesis sobre la estructura gra- matical de la protolengua, pues los otros dos se concentraron en lo fonológico de manera exclusiva. Las propuestas de Wheeler, relativas precisamente a presuntos elementos clasificadores, pueden considerarse, sin embargo, carentes de validez, debido a las deficiencias que falsean las reconstrucciones sobre las que se hicieron (Constenla Umaña 1983: 42-6; Holt 1986: 70).

Este artículo intentará hacer una contribución a este aspecto de la lingüística chibcha todavía tan poco desarrollado, partiendo de las bases que en materia fonológica y léxica se dan en mi estudio de 1981.

Su propósito es aportar indicios comprobadores de la existencia de clasificadores en el protochibcha. Concretamente, se plantea la hipótesis de que los étimos *kuā 'semilla' y *karz̄ 'hueso, palo' se emplearon en dicha lengua como clasificadores de los elementos nominales en el nivel sintáctico (esto es, como componentes directos de los sintagmas nominales), no a nivel morfológico (como afijos en los numerales, que es la situación prevaleciente en las lenguas descendientes).

\section{Reflejos de *kuā como sufijo}

*kuā ha dado origen, en la mayor parte de las lenguas, a sufijos empleados en la derivación de sustantivos. En algunas de ellas aparecen también 
reflejos suyos como derivativos de adjetivos y numerales.

1.1. En dobocubí, el sufijo - $k w a$ aparece con mucha frecuencia en los datos del siglo XVIII (Rivet y Armellada 1950), no así en los recogidos en el presente siglo. Se presenta como derivativo de sustantivos y adjetivos por igual.

Ejemplos (2):

$\begin{array}{llll}\text { dokwa } & \text { 'rostro', } & \text { talikwa } & \text { 'blanco' } \\ \text { borokwa } & \text { 'plátano' } & \text { itakwa } & \text { 'caliente' } \\ \text { očokwa } & \text { 'rodilla', } & \text { terokwa } & \text { 'frío' } \\ \text { tankwa } & \text { 'maraca' } & \text { vitrunkwa } & \text { 'verde' }\end{array}$

1.2. Reichel-Dolmatoff (1947: 25-6) incluye el sufijo - $k v a$ (escrito - $k u a$ en los datos de Celedón 1886) entre los más comunes en la derivación de sustantivos en chimila.

Ejemplos:

$\begin{array}{llll}\text { guákva } & \text { 'ojo' } & \text { kákva } & \text { 'boca' } \\ \text { kókva } & \text { 'dedo' } & \text { núkva } & \text { 'muslo', } \\ \text { tákva } & \text { 'guacal' } & \text { uákva } & \text { 'mofeta' }\end{array}$

1.3. En tunebo, el sufijo - $k w a$ se presenta con cierta frecuencia en la formación de sustantivos.

Ejemplos:

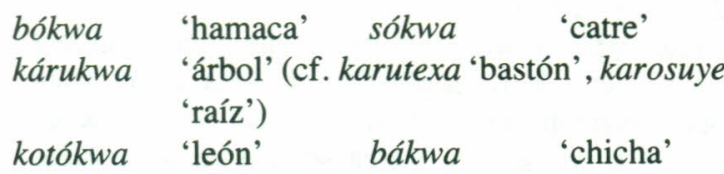

1.4. En cágaba, Preuss (1925: 884) incluye el sufijo - kua entre los que derivan temas sustantivos $\mathrm{y}$ adjetivos. Este sufijo presente -gua como variante en posición intervocálica y se da también en la formación de numerales.

Ejemplos:

malkua 'araña' múlkua 'blanco'
máigua 'tres'

múgua 'chile' (múkua para Celedón 1886:48)

1.5 Las otras lenguas aruacas, para las que no existen todavía estudios de morfología derivativa, presentan los sufijos bíntucua - $k w i$ (Frank 1985; $-k u e ̈$ en Vinalesa 1952), guamaca -kwa y atanques $-k u a$, como equivalentes del cágaba -kua.
Ejemplos:

$\begin{array}{llll} & \text { bíntucua } & \text { guamaca } & \text { atanques } \\ \text { araña } & \text { mánkwi } & \text { mínkwa } & \text { mánkua } \\ \text { siete } & & \text { kúgwa } & \\ \text { tres } & & \text { máigwa } & \end{array}$

llaga soukuë (cf. cágaba sáuña 'llaga', forma que presenta la misma raíz)

1.6. En muisca - cua (precedido por /a/, /o/ y consonantes no siseantes) у -рсиа (precedido por /e/, /i/, /u/, / / / y consonantes siseantes) se presenta en la derivación de diversos tipos de temas, entre ellos sustantivos y adjetivos. Entre otros temas en que aparece se encuentran un numeral y dos interrogativos.

Ejemplos:

chupcua 'pezón' (chue 'teta') cocua 'punta del dedo' quichcua 'cuello del pie' (quihicha 'pie')

cuhupcua 'sordo' (cuhuca 'oreja') chitupcua 'caliente' cuhupcua 'siete' (cf. cuhuna 'hace siete días')

ipcua 'qué' ficua 'cuántos'

1.7. El sufijo cuna - $k w a$ aparece tanto en sustantivos como en adjetivos y numerales de acuerdo con Holmer (1947:54-6). En varios casos, la forma con sufijo alterna con la integrada sólo por la raíz.

Ejemplos:

$\begin{array}{ll}\text { akkwa } & \begin{array}{l}\text { 'piedra' talakwa 'ojo' (en variación con } \\ \text { tala) }\end{array} \\ \text { winkwa } & \begin{array}{l}\text { 'cuenta' (cf. wini 'cuentas') wakwa 'nieto' } \\ \text { kolokwa 'amarillo' sipukwa 'blanco' (en variación con } \\ \text { sipu) }\end{array} \\ \text { pokwa 'dos' (la raíz poo se emplea sola con ciertos sus- } \\ \text { tantivos) } \\ \text { nerkwa 'seis' pikwa 'cuánto' }\end{array}$

1.8. En dorasque se presenta un sufijo $-k u$ (escrito también - $g u$, pues no había oposición entre $k$ y $g$ ) como derivativo de sustantivos que en otras lenguas presentan cognados construidos con reflejos de *kuā:
negú 'nariz' (cf. térraba 'nekwo' 'nariz')
dukú 'cabeza' (cf. movere dokws 'cabeza'; dobocubí dokwa 'rostro')

También aparecen formas derivadas por medio de -kua (o -gua), que podría ser una variante del mismo morfema que - $k u$ (la pérdida de $a$ final de morfema es frecuente en este idioma). 
Ejemplos:

kagua 'labios' (cf. chimila kákva 'boca')

kulgua 'uña' (cf. kulá 'mano')

sotkua 'mojarrablanca' (cf. boruca sō't 'mojarra')

1.9. Los materiales léxicos disponibles en el caso del bocotá son escasos; sin embargo, en los temas sustantivos se presenta con frecuencia $-k w a$ como sufijo derivativo.

Ejemplos:

bogakwa 'nube' (en variación con boga 'idem')

holókwa 'saliva' skugékwa 'murciélago'

skígwa 'pulga' búkwa 'artesa en forma de hamaca'

1.10. En movere, $-k w$ s es un sufijo derivativo frecuente en la formación de sustantivos.

Ejemplos:

$\begin{array}{lll}\text { nukws 'ave' (cf. bribri } d \bar{u} \text { 'ave') } & \text { brukws 'corazón' } \\ \text { nokws 'fuego' dokws } & \text { 'cabeza' } \\ \text { munkws 'mosca' kusokws 'puño' }\end{array}$

1.11. Desde el estudio de Pittier y Gagini (1892:10), -kuo ha sido reconocido como derivativo de sustantivos frecuente en térraba.

Ejemplos:

bokuo ‘ojo' (cf. borio 'lágrima')
orkuo 'mano' nekuo 'nariz'
khokuo 'cielo' 'Фiskuo 'gato' cf. boruca bis 'gato'

1.12. En boruca, -kua se presenta en la derivación de cierto número de sustantivos.

Ejemplos:

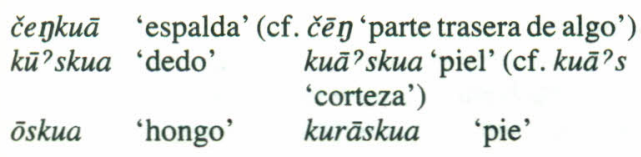

1.13. En rama no se ha observado sino una forma que parezca contener un reflejo de *kuā como sufijo: tǔkǔà 'muslo' (cf. bribri tú? , chimila núkva 'muslo').

1.14. En paya se ha observado un sustantivo terminado en -wa, sawa 'mano', que podría estar emparentado con el térraba sákuo 'dedo', formado por medio del sufijo $-k u o(<* k u a \overline{)}$. El sufijo -wa aparece en algún otro sustantivo, pero su presencia es mucho más frecuente en los adjetivos. En los numerales ordinales también podría estar presente este elemento como parte de la pauta de formación que incluye simultáneamente la presencia de un prefijo ãwh-. -wa sería el retroflejo esperado de * kuā en paya, lengua en la que un cambio fonológico ha suprimido la $* k$ inicial (Constenla Umaña 1981:250).

Ejemplos:

\begin{tabular}{|c|c|}
\hline aso:wa & 'río' (cf. aso: 'agua') \\
\hline sẽwa, & 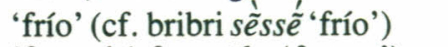 \\
\hline atapứhwa & 'fuerte' (cf. tapũha 'fuerza') \\
\hline patawkwa & 'caliente' (cf. patawkka 'calor') \\
\hline ãwhpókwa & ‘segundo’ (cf.pok'dos’) \\
\hline$\tilde{a} w h m \tilde{a} y h w a$ & 'tercero' (cf. mãyh 'tres') \\
\hline
\end{tabular}

\section{Reflejos de *karð como sufijo}

* karð ha dado origen, en la mayor parte de las lenguas a sufijos empleados en la derivación de sustantivos. En algunas de ellas aparecen también reflejos suyos como derivativos de adjetivos y numerales.

2.1. Reichel Dolmatoff (1947:26) incluye $-k r a$ entre los sufijos más frecuentes en la derivación de sustantivos en chimila.

Ejemplos:

krangkra 'araña' mukra 'cola'
háta:kra 'mano' (cf. tunebo átkara 'mano')
úkra
kíkra 'cara' (cf. tunebo úkara'frente')
'hueso' mitakra 'pecho'

2.2. Rivet (1924:31) incluye -kara entre los derivados de sustantivos más frecuentes en tunebo.

Ejemplos:

ríkara 'día' (cf. ríbitro 'hace sol')

káikara 'hueso' késkara 'pie'

úkara 'frente' kuskara 'rodilla'

síkara 'aguja' átkara 'mano'

2.3. Preuss (1925:883) incluye -kala (escrito $-k a l l a$ y $-k \Lambda l d \Lambda \sim x \Lambda l d \Lambda$ en otras fuentes) entre los derivativos de sustantivos en cágaba.

Ejemplos:

kaukalla 'mano' (la raíz kau- proviene del mismo étimo que la del chimila kókva 'dedo')

nikalla 'lluvia' (cf. $n i$ 'agua')

mulkslds 'viento' (cf. rama pứlkăt 'viento')

zalakala 'hueso' tabakala 'pecho' 
En la misma lengua, al lado de la serie de numerales en -kua (véase 1.4.), Preuss (ibidem: 893) señala la exístencia de otra en -kala, si bien no deja en claro cuál es la diferencia entre ambas; de modo que se encuentran formas como:

maigua, maikala 'tres'

kugua, kukala 'siete'

2.4. En las otras lenguas aruacas *karz̄ tiene como reflejos sufijados el bínticua -kıni (escrito también -kana, -kına), el guamaca -kuna (escrito también -khəna, -kənə) y el atanques -kana (escrito también -kane, -köna).

Ejemplos:

\begin{tabular}{|c|c|c|c|}
\hline $\begin{array}{l}\text { nariz, hocico } \\
\text { ala } \\
\text { muslo } \\
\text { cola }\end{array}$ & $\begin{array}{l}\text { bíntucua } \\
\text { tunkana }\end{array}$ & $\begin{array}{l}\text { guamáa } \\
\text { sunkúna } \\
\text { gwukkúna } \\
\text { dukukkhóna } \\
\text { duškənə }\end{array}$ & $\begin{array}{l}\text { atanques } \\
\text { sunskane } \\
\text { kuichikane } \\
\text { dukökana } \\
\text { duchikána }\end{array}$ \\
\hline $\begin{array}{l}\text { rodilla } \\
\text { angosto } \\
\text { huso }\end{array}$ & $\begin{array}{l}\text { biukর́ni } \\
\text { inguikana } \\
\text { kúrkana }\end{array}$ & & \\
\hline
\end{tabular}

En los numerales, se desconoce si, al lado de la serie terminada en reflejos de $* k u \bar{a}$, existe otra en guamaca y atanques. La única serie del bíntucua, de acuerdo con Frank (1985:40-1), presenta en la mayor parte de los casos reflejos de $* k u \bar{a}$, pero hay un numeral cuyo sufijo es reflejo de *karz̃: máikıni 'tres' (cf. ma'keiwa 'cuatro', asewa 'cinco' y el cágaba máigua 'tres').

2.5. Adam (1878:32) incluye -quyn entre los principales sufijos de la flexión nominal en muisca.

Ejemplos:

$\begin{array}{ll}\text { fizequyn } & \text { 'gaznate' (cf. fiza 'cuello') } \\ \text { gutaquyn } & \text { 'parte inferior de la espalda' } \\ \text { iomguyn } & \text { 'tobillo' } \\ \text { quichcuaquyn } & \text { 'calcañar' (cf. quihicha 'pie') } \\ \text { sitiquyn } & \text { 'espinazo' tobiaquyn 'costillas' }\end{array}$

2.6. En cuna, se presenta -kala ( -kal o -kar) como derivativo de sustantivos, si bien con una frecuencia muy baja (Holmer 1947: 65).

Ejemplos

nukála 'diente' (cf. Ou 'diente', Oukála ‘muela', en dorasque)

pinaskala 'pecho' tukkala 'cuello'
2.7. En dorasque y chánguena, -kala $(\sim-k a l$ o -gala,) aparece como derivativo de sustantivos.

Ejemplos:

$\begin{array}{lll}\text { asiento } & \begin{array}{l}\text { dorasque } \\ \text { sérkala }\end{array} & \begin{array}{l}\text { chánguena } \\ \text { sérkala }\end{array} \\ \text { calavera } & \text { kikála } & \\ \text { hombro } & \text { kirkal } & \\ \text { costilla } & \text { (sikli) crikrigala } \\ \text { muela } & \text { Oukála (cf. Ou 'diente') } \\ \text { nube } & \text { bokala }\end{array}$

2.8. En bocotá, - gda es un derivativo de sustantivos frecuente.

Ejemplos:

seragda 'pierna' (cf. sera 'pie')
skugda 'cola', bligda'comida' (cf. bli 'comer')
trigda 'espalda' higda 'fuego'

2.9. El reflejo movere - $k r a$ es un derivativo de sustantivos muy frecuente.

Ejemplos:

tr $x k r \supset$ 'espalda' (en variación con la palabra integrada por la raíz sola: $\operatorname{tr} \gamma$ )

hukro 'armazón de la casa' ( $h u$ 'casa')

kskro 'bejuco'

okro 'frente' (cf. okws 'ojo')

skro 'semilla' de yuca' (cf. 8 'yuca')

2.10. El reflejo boruca, $-k r a \sim-k r a \bar{a}$, es uno de los derivativos de sustantivos más frecuentes de la lengua.

Ejemplos:

\begin{tabular}{|c|c|c|}
\hline baxkrā & 'caña blanca' & 'flecha' \\
\hline mankrā & ‘cuchillo’' & 'hueso' \\
\hline ǰkrā & 'fuego' (cf. & 'leña') \\
\hline$s \bar{u}^{?} k r a$ & 'vena' & $\begin{array}{l}r a \text { 'jobo (árbol)' (cfr. brā? } u \\
\text { del jobo') }\end{array}$ \\
\hline
\end{tabular}

2.11. En cabécar, -klø se presenta en un número reducido de sustantivos.

Ejemplos:

$k s \bar{\varnothing} k \grave{l} \oplus$ 'isogro’ (chamán que cantaba en los funerales; de $k s \omega$ 'cantar')

hĩdẫklø 'dinero' (de hĩdã 'jugar')

diklø 'agua, río' (*dí? 'agua') 
Un numeral, $\bar{e} k \grave{l} \bar{a}$ 'uno (humano)', presenta -kla en lugar de -klø como reflejo de *karā. No se tiene una explicación fonólogica de este cambio de vocal, pero es una pauta de la lengua que los sufijos clasificadores de los numerales presenten $a$ con la raíz $e$ - 'uno' y $₫$ con las demás raíces: $\bar{e} t k \bar{a}, b \bar{\oplus} t k \bar{\omega}$, bajjẫ̂tkळ 'uno, dos, tres... (cosas cilíndricas)'.

2.12. En bribri, el reflejo de *karā como sufijo parece ser $-k \bowtie l$, si bien la forma esperada sería más bien -kal. No obstante, la comparación con el cabécar indica que el bribri llevó a cabo procesos semejantes de síncopa y metátesis en otros casos; por ejemplo, el cabécar b̄̄tabø 'dos (cósas cilíndricas)' es $b \bar{\varnothing} t \omega b$ en bribri. Por el momento, no se han determinado las condiciones fonológicas que intervinieron en estos procesos. $-k \bowtie l$ se presenta en un número reducido de sustantivos y en un numeral.

\section{Ejemplos:}

\begin{tabular}{|c|c|}
\hline 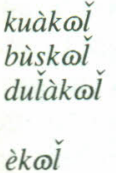 & 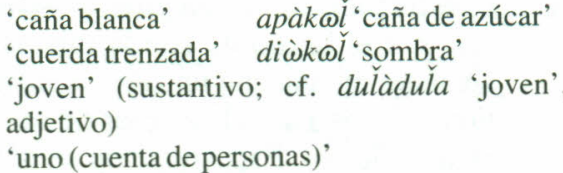 \\
\hline
\end{tabular}

2.13. En térraba, -ǧ̆o se presenta con frecuencia como derivativo de sutantivos.

Ejemplos:

$\begin{array}{ll}\text { 'sroglo } & \text { 'caña de bambú' (en variación con la forma } \\ & \text { integrada por la raíz sola: sro) } \\ \text { 'kønglo } & \text { 'flecha' do'boglo 'hueso' } \\ \text { 'boglo } & \text { 'frente' di'oglo 'alma, espíritu' } \\ \text { 'th@rglo } & \text { 'juguete'(cf. thør 'jugar') }\end{array}$

2.14. En guatuso, no hay ningún sufijo que sea reflejo de *kar̄̄, pero si una palabra de significado muy general ko:ra 'árbol, palo, hueso, objeto largo y cilindríco' que se emplea en frases que equivalen aproximadamente al tipo de formas con sufijos de las otras lenguas.

Ejemplos:

\section{kuki: ko:ra 'brazo' (kuki: 'extremidad superior') \\ ča: ko:ra 'calavera' (ča: 'cabeza')}

En algún caso estas frases se han fosilizado, al dejar de funcionar el primer elemento independientemente, de modo que actualmente han pasado a constituir una sola palabra, como sucede en $k a \bar{r}$ ko:ra 'muslo' (cuyo primer elemento aparente- mente se presenta reduplicado en kar̄kāra: 'hombro').

2.15. En rama, -kat se presenta en la formación de numerosos sustantivos y algún adjetivo.

Ejemplos:

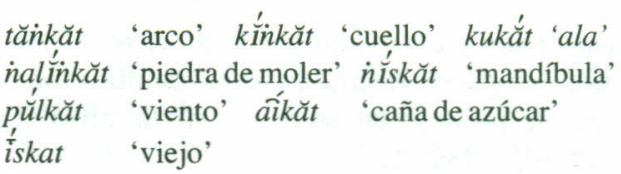

\section{Reflejos de *kuā como prefijo}

En cuatro lenguas - cuna, dorasque, movere y térraba- se dan prefijos derivados de *kuā. En los cuatro casos se trata de clasificadores que aparecen con las raíces de los numerales.

3.1. En cuna (Holmer 1947:101) -kwa se presenta como prefijo clasificador en numerales referidos a cosas más o menos redondas (frutas, pepitas de oro), casas y algunas aves.

Ejemplo:

sappi turpa $k w a-k w e n$
árbol fruta clas.-una

Holmer (idibem) consideró que este prefijo era, probablemente, reflejo del mismo étimo que el sufijo - $k w a$ de la lengua (véase 1.7.).

3.2. En dorasque se dio una serie de numerales iniciada por un prefijo $k u$ - (véase 1.8.), si bien se desconoce a cuáles objetos se aplicaba:

ku-e 'uno', ko-mo 'dos', ku-mas 'tres', ku-paki 'cuatro', ku-lmale 'cinco'.

3.3. En movere, $k w a$ - (ante la raíz 'uno') $\sim k u$ (ante la raíz 'dos') ko- (ante las raíces 'tres' y 'cuatro') $\sim k w o$ - (en los demás casos) es el clasificador de los numerales en la cuenta de, entre otras cosas, frutas redondas, aves, ollas, casas y piedras:

$k w a-t i$ 'uno', ku-bu 'dos', ko-mo 'tres', ko-boks 'cuatro', kwo-rike 'cinco', kwo-ti 'seis', kwo-kuku 'siete', etc.

3.4. En térraba khua- (ante la raíz 'uno') khuo(en los demás casos) se presenta como clasificador en los numerales y en algunos adjetivos, como -tira 
'pequeño' cuando se emplean referidos a, por ejemplo, frutas redondas, piedras, semillas, aves, insectos y objetos redondos en general:

khua-'ra 'uno', khuo-'bø 'dos', khuomî̀ $\tilde{a}$ 'tres', etc. khuo-'tira 'pequeño'

La aspiración presente en los alomorfos del prefijo de esta lengua es resultado de un cambio fonológico que se produjo en posición inicial absoluta (cf. Constenla 1981: 227).

\section{Reflejos de *karə̄ como prefijo}

En cuatro lenguas - dorasque, bocotá, movere y térraba - se dan prefijos derivados de *kar̄̄. En los cuatro casos se trata de clasificadores que se usan con las raíces de los numerales.

4.1. En dorasque se dio una serie de numerales iniciada por kala-, si bien no han llegado hasta nosotros detalles de su uso. Ejemplos de la misma son kala-bai 'tres' y kala-paka 'cuatro'.

4.2. En bocotá se da una serie iniciada por $g d a$ que se emplea para contar árboles, objetos cilíndricos como cuerdas, reptiles, mamíferos y personas:

gda-ite 'uno', gda-boke 'dos', gda-mai 'tres'

4.3 En movere kra- (ante la raíz 'uno') kro(ante la raíz 'dos') kro- (en los demás casos) es el prefijo que se da con los numerales referidos a cosas cilíndricas como troncos de árbol, frutos de musáceas, culebras, ríos y también a cuadrúpedos:

kra-ti 'uno', kro-bu 'dos', kro-mo 'tres', kro-boks 'cuatro'

4.4. En térraba, khla- (ante la raíz 'uno') khlo(en los demás casos) se usa con las raíces de los numerales y algunas adjetivas, como -tira 'pequeño' cuando éstos se refieren a objetos de forma más o menos cilíndrica, reptiles, mamíferos y personas:

khla-'ra 'uno', khlo-'bø 'dos', khlo-mî̀ $\tilde{a}$ 'tres'

5. Resumen sobre el uso de reflejos de $* k u \bar{a}$ 'semilla' y *karð̄ 'hueso, palo' como afijos.
Los datos examinados en las secciones precedentes nos señalan los siguientes hechos interesantes sobre el uso de reflejos de *kuā 'semilla' y *karā 'hueso, palo' como afijos en las lenguas chibchas:

a. Los reflejos sufijados de estos elementos, en la mayor parte de las lenguas, funcionan como derivativos de sustantivos y, en algunas, de adjetivos y numerales también.

b. Semánticamente, entre los sustantivos derivados por medio de reflejos de *karā se nota una tendencia al predominio de los referidos a objetos alargados y cilíndricos y, entre los derivados por medio de reflejos de $* k u \bar{a}$, al de los referidos a objetos más o menos redondos, circulares o a extremos no puntiagudos. Esto, por supuesto, resulta más obvio en el caso de las lenguas que presentan reflejos de los dos étimos.

c. Con frecuencia, las lenguas presentan diferencias por el hecho de que la misma raíz pueda aparecer en una con uno de los sufijos en cuestión y en otra sin él, a pesar de ser uno mismo el significado. Esta situación se da también en una misma lengua en muchos casos.

ch. El uso de reflejos de los dos étimos aludidos como prefijos está restringido a lenguas habladas en época precolombina en el actual territorio panameño (cuna, dorasque, bocotá, movere y térraba). En todas ellas, estos elementos se presentan como prefijos clasificadores de numerales, en general, con un valor semejante al señalado para su uso como derivadores de sustantivos.

\section{Conclusiones}

La hipótesis que se plantea, a partir de los hechos que se acaban de resumir, es que la protolengua empleó estas formas ( $\mathrm{y}$, probablemente, otras, como se verá luego) como clasificadores nominales.

Estos elementos habrían sido, en aquel estadio de la evolución, no afijos, sino palabras independientes, colocadas probablemente después del sustantivo.

La existencia de reflejos tanto prefijados como sufijados de los dos étimos en modificadores del sustantivo (numerales y adjetivos) en lenguas descendientes hace necesario proponer dos órdenes 
facultativos en el sintagma nominal de la protolengua: sustantivo + modificador + clasificador $y$ sustantivo + clasificador + modificador.

Aún en el caso de las lenguas que han conservado el valor clasificador de los dos étimos, empleándolos como afijos en los numerales, las apariciones de éstos en ciertas secuencias tienen que haber ido perdiendo gradualmente el valor clasificador. Dichas secuencias habrían sido, para casi todas las lenguas, aquellas en las que el modificador era un adjetivo (sólo una lengua de las que se han tomado en cuenta en este estudio, el térraba, emplea clasificadores con un número pequeño de palabras de este tipo).

En muchas lenguas, el valor clasificador de $* k u \bar{a}$ y *karā llegó a perderse totalmente, esto es, incluso en las construcciones con numerales. En algunos casos, esta pérdida debe de haber sucedido tardíamente, como lo sugiere, en el caso del bíntucua, la presencia de ambos étimos como sufijos en la única serie de numerales que se da actualmente.

La pérdida, total o parcial, del valor clasificador debe de haber permitido la reinterpretación de las secuencias sustantivo + clasificador como integrantes de una sola palabra, lo cual habría dado origen al uso de los elementos en cuestión como sufijos derivativos de sustantivos. Como todo sustantivo habría podido originariamente aparecer seguido o no por los clasificadores, cualquiera de las dos formas aislada o la seguida por el clasificador) habría podido ser escogida como punto de partida. Esto explicaría por qué para una misma noción se puede encontrar tanto un tema formado por medio de una raíz sola como por la misma raíz en secuencia con un reflejo de $* k u \bar{a}$ o ${ }^{*} k a r \bar{\partial}$, aun en una misma lengua.

Anteriormente se señaló que además de los étimos discutidos debieron de existir otros que fueran empleados como clasificadores sintáticos en la protolengua. Tal podría haber sucedido en el caso del prefijo clasificador de objetos planos del movere (ka- ko- ko-) y del bocotá (ga-), evidentemente relacionado con * $k$ ə 'hoja' (>movere $k s$, bocotá - $g a$ ), que se encuentra en cuna ( $k a-$, Holmer 1947:101), referido a peces, pero también a lápices, cigarrillos y escopetas. Este caso, sin embargo, necesita mayor estudio, pues además de la dificultad semántica planteada por el cuna, no parece haber huellas de $* k \bar{a}$ como afijo en la mayor parte de las lenguas, si bien en guatuso hay un sufijo $-k a$ que entra en la formación de diversos temas cuantificadores (como paunka 'dos' y pinka 'cuán- tos'), y en bíntucua y atanques hay algunos numerales en -ga: móuga 'dos', koga 'siete', úga 'diez' (bíntucua), móga 'dos', kuga 'siete' (atanques).

Finalmente, es interesante que sean los clasificadores de las formas redonda, alargada y plana (en especial, los de las dos primeras) los que parecen poderse reconstruir en este caso. Clark y Clark (1978: 239-40) ha señalado que en las lenguas que los presentan los clasificadores tienden a basarse fundamentalmente en la forma y que las formas más frecuentemente escogidas son las mencionadas. De acuerdo con ellos, dichas formas son simples desde el punto de vista de la percepción y precisamente las dos primeras son las que los niños de manera universal en sus clasificaciones:

Los niños parecen encontrar que algunas formas se destacan más que otras y las usan en sus primeras hipótesis en materia de selección de sustantivos.

Los nombres de estas formas, por lo tanto, se aprenderán con facilidad y con la predilección de los adultos por las mismas formas, también se mantendrán en la lengua. Los nombres básicos de las formas, entonces, están determinados por principios que forman parte del sistema perceptivo humano.

\section{NOTAS}

(1) El presente artículo fue escrito como parte de las actividades de investigación realizadas durante mi licencia sabática con el apoyo de la Universidad de Costa Rica y la Universidad Estatal de Nueva York en Albany.

Algunos de los datos de las lenguas aruacas y el tunebo provienen de listas facilitadas por Paul Frank del Instituto Lingüístico de Verano de Colombia. Los datos del paya provienen de materiales inéditos de Dennis Holt que me fueron facilitados por Lyle Campbell.

Mi gratitud tanto para las personas como para las instituciones mencionadas.

(2) Los datos aparecen transcritos como en las obras de las cuales se tomaron. Fuera de las citadas en el artículo y en la nota precedente, se consultaron las siguientes: Uricoechea 1871; Celedón 1886, 1890a, 1890b; Pinart 1890; Lehmann 1920, Vinalesa 1952, Levinsohn 1975.

En el caso del bribri, el cabécar, el boruca, el térraba y el guatuso he empleado mis propios datos transcritos de acuerdo con los principios del Alfabeto Fonético Internacional, excepto por lo que respecta a la lateral vibrante, las vibrantes centrales simple y múltiple, y las africadas prepalatales sorda y sonora, que se han transcrito con los símbolos [ $\mathrm{l}],[\mathrm{r}],[\overline{\mathrm{r}}],[\mathrm{c}],[\breve{\mathrm{j}}]$, respectivamente.

\section{BIBLIOGRAFIA}

Adam, Lucien. 1878. Études sur six langues américaines: Dakota, Chibcha, Nahuatl, Kechua, Quiché, Maya. París. 
Beuchat, Henri y Paul Rivet. 1910. "Affinités des langues du Sud de la Colombie et du Nord de l'Equateur". Le Mouséon 11: 33-68, 141-98.

Celedón, Rafael. 1886. Gramática de la lengua köggaba, con vocabularios y catecismos. París: Maisonneuve.

. 1892a. "Vocabulario de la lengua atanques". Actas del VIII Congreso Internacio-" nal de Americanistas: 591-9.

. 1892b. "Vocabulario de la lengua bintucua". Actas del VIII Congreso Internacional de Americanistas: 599-609.

Clark, Eve y Herbert H. Clark. 1978. "Universals, Relativity and Language Processing “. En: Universals of Human Language, J. H. Greenberg (compilador), Vol. 1 Method and Theory: 225278. Standford: Standford University Press.

Constenla Umaña, Adolfo. 1981. "Comparative Chibchan Phonology". Tesis doctoral. Universidad de Pensilvania.

. 1983. "Desarrollo del estudio diacrónico de las lenguas chibchas". Estudios de Lingüística Chibcha 2: 15-66.

Conzemius, Edward. 1929. "Die Rama-Indianer von Nicaragua". Zeitschrift für Ethnologie 59: 291-362.

Frank, Paul S. 1985. "A grammar of Ika". Tesis doctoral. Universidad de Pensilvania.

Greenberg, Joseph H. 1987. Language in the Americas. Standford: Standford University Press.

Holmer, Nils M. 1947. "Critical and Comparative Grammar of the Cuna Language". Etnologiska Studier 14. Gotemburgo.

Holt, Dennis. 1986. "The Development of the Paya Sound System”. Tesis doctoral. Universidad de California, Los Angeles.

Lehmann, Walter. 1920. Zentral Amerika, teil I, Die Sprachen Zentral-Amerikas in ihren Beziehungen zueinander sowie zu Süd-Amerika und Mexiko. Berlín: Dietrich Reimer.
Levinsohn, Stephen H. 1975. "El bokotá, el guaymí y el teribe respecto al proto-chibcha". En: Lenguas de Panamá II. Observaciones preliminares sobre los sistemas gramaticales de las lenguas chibchas: 3-18. Panamá: Instituto Lingüístico de Verano e Instituto Nacional de Cultura.

Lévi-Strauss, Claude. 1948. "Sur certaines similarités des langues Chibcha et Nambikwara". Actas del XXVIII Congreso Internacional de Americanistas: 185:92.

Pinart, Alphonse L. 1890. Vocabulario CastellanoDorasque, dialectos Chumulu, Gualaca y Changuina. París: Leroux.

Pittier, Henri F. y Carlos Gagini. 1892. Ensayo lexicográfico sobre la lengua Térraba. San José.

Preuss, Konrad T. 1925. "Forschungsreise zu den Kágaba-Indianern der Sierra Nevada de Santa Marta in Kolumbien”. Anthropos 20: 881-928.

Reichel Dolmatoff, Gerardo. 1947. "La lengua chimila". Journal de la Société des Américanistes de París 36: 15-50.

Rivet, Paul. 1924. "La langue Tunebo". Journal de la Société des Américanistes de Paris 16: 19-92.

Rivet, Paul y Cesáreo de Armellada. 1950. "Les Indiens Motilones". Journal de la Société des Américanistes de Paris 39: 15-57.

Uricoechea, Ezequiel. 1871. Gramática, vocabulario, catecismo i confesionario de la lengua Chibcha, según antiguos manuscritos anónimos e inéditos, aumentados y corregidos. París:Maisonneuve.

Vinalesa, José de. 1952. Indios arhuacos de la Sierra Nevada de Santa Marta, descripción geográfica, costumbres de los indios, idioma arhuaco. Bogotá: Editorial Iqueima.

Wheeler, Alva L. 1972. "Proto-Chibchan". En: Comparative studies in Amerindian Languages. Esther Matteson (compiladora): 96-111. La Haya: Mouton. 